(C) World Scientific Publishing Co. \& Operational Research Society of Singapore DOI: $10.1142 / \mathrm{S} 0217595910003009$

\title{
AUTHOR INDEX \\ Volume 27
}

Aghaei, J., Shayanfar, H. A. \& Amjady, N., Stochastic

Market-Clearing of Joint Energy and Reserves Auctions

5 (2010) 587

Alirezaee, M. R. \& Shiraz, R. K., A Note on an Extended

Numeration Method for Solving Free Disposal Hull Models in $D E A$

5 (2010) 607

5 (2010) 587

Amjady, N., see Aghaei, J.

Antczak, T., The $l_{1}$ Penalty Function Method for Nonconvex

Differentiable Optimization Problems with Inequality

Constraints

5 (2010) 559

Artalejo, J. R. \& Resing, J. A. C., Mean Value Analysis of Single Server Retrial Queues

3 (2010) 335

Asad, M. W. A., see Rehman, S. U.

3 (2010) 315

Basu, M., see Saha, S.

3 (2010) 393

Ben-Daya, M., see El-Ferik, S.

4 (2010) 539

Beraldi, P., Ghiani, G., Musmanno, R. \& Vocaturo, F., Efficient

Neighborhood Search for the Probabilistic Multi-Vehicle Pickup and Delivery Problem

3 (2010) 301

Cai, X., see Li, Y.

3 (2010) 411

Cai, X., see Li, Y.

5 (2010) 629

Cao, Z.-G., see Zhang, S.-X.

4 (2010) 493

Chakravarthy, S. R., Dudin, A. N. \& Klimenok, V. I., A Retrial Queueing Model with Map Arrivals, Catastrophic Failures with Repairs, and Customer Impatience

6 (2010) 727

Chang, J.-Y., see Yu, J. C. P.

4 (2010) 437

Chang, K.-L., see Liao, S.-K.

6 (2010) 753

Chen, J., see Li, Y.

3 (2010) 411

Chen, J., see Li, Y.

5 (2010) 629

Chen, M.-S. \& Chen, Y.-C., A Note on the Optimal Supply

Cycle When the Whole Period is Stockout

5 (2010) 611

Chen, Y.-C., see Chen, M.-S.

5 (2010) 611

Cheng, M.-B., see Wang, W.-W.

5 (2010) 617

Currie, C. S. M., see Lu, L.

1 (2010) 121

Dahiya, K., see Sharma, V.

4 (2010) 457 
Dai, Y.-H., see Fu, Y.-S.

1 (2010) 71

Dudin, A. N., see Chakravarthy, S. R.

6 (2010) 727

El-Ferik, S. \& Ben-Daya, M., Integrated Production Maintenance Model Under Imperfect Age-Based Maintenance Policy and Non-Negligible Maintenance Times

Fu, R., see Lin, L.

Fu, Y.-S. \& Dai, Y.-H., Improved Projected Gradient Algorithms for Singly Linearly Constrained Quadratic Programs Subject to Lower and Upper Bounds

Ghiani, G., see Beraldi, P.

González-Martín, C., see Sedeño-Noda, A.

Goto, M., see Takashima, R.

He, C., Lin, Y. \& Yuan, J., Some Improved Algorithms on the Single Machine Hierarchical Scheduling with Total Tardiness as the Primary Criterion

He, L.-M., see Wang, W.-W.

Hung, S.-W., see Pan, T.-W.

Ibaraki, T., see Nishihara, M.

4 (2010) 539

6 (2010) 669

1 (2010) 71

3 (2010) 301

6 (2010) 677

2 (2010) 271

Ito, D., Ohnishi, M. \& Tamba, Y., Pricing and Calibration of a Chooser Flexible Cap

Kawai, K.-I., see Lu, X.

Kimura, T., Alternative Randomization for Valuing American Options

5 (2010) 577

5 (2010) 617

3 (2010) 369

2 (2010) 211

2 (2010) 243

2 (2010) 287

2 (2010) 167

6 (2010) 727

2 (2010) 257

Ko, C.-C., see Lin, T. T.

1 (2010) 15

1 (2010) 1

Konno, H., see Takaya, Y.

Kunita, H. \& Yamada, T., Average Options for Jump Diffusion Models

2 (2010) 143

6 (2010) 649

Li, S., see Sun, W.

Li, Y., Chen, J., Cai, X. \& Tu, B., Optimal Manpower Planning Decision with Single Employee Type Considering Minimal Employment Period Constraint

3 (2010) 411

Li, Y., Zhang, J., Chen, J. \& Cai, X., Optimal Solution Structure for Multi-Period Production Planning with Returned Products Remanufacturing

5 (2010) 629

Liao, S.-K., Chang, K.-L. \& Tseng, T.-W., Optimal Selection of Program Suppliers for TV Companies Using an Analytic Network Process (ANP) Approach

6 (2010) 753

Lin, L., Lin, Y., Zhou, X. \& Fu, R., Parallel Machine Scheduling with a Simultaneity Constraint and Unit-Length Jobs to Minimize the Makespan 
Lin, T. T., Ko, C.-C. \& Liu, C.-Y., An Optimal Market

Entry/Exit Evaluation Model with Partial Financing Funds

2 (2010) 257

Lin, Y., see He, C.

5 (2010) 577

Lin, Y., see Lin, L.

6 (2010) 669

Liu, C.-Y., see Lin, T. T.

2 (2010) 257

Lu, L. \& Currie, C. S. M., Evaluation of the Arrows Method for Classification of Data

1 (2010) 121

3 (2010) 369

Lu, W.-M., see Pan, T.-W.

Lu, X., Kawai, K.-I. \& Maekawa, K., Estimating Bivariate GARCH-Jump Model Based on High Frequency Data: The Case of Revaluation of the Chinese Yuan in July 2005

2 (2010) 287

Maekawa, K., see Lu, X.

2 (2010) 287

Mevissen, M. \& Kojima, M., SDP Relaxations for Quadratic Optimization Problems Derived from Polynomial Optimization Problems

1 (2010) 15

3 (2010) 301

Musmanno, R., see Beraldi, P.

Ni, Q., see Wang, H.-J.

1 (2010) 85

Nishihara, M., Yagiura, M. \& Ibaraki, T., Computing Bounds on Risk-Neutral Distributions from the Observed Prices of Call Options

2 (2010) 211

2 (2010) 243

Özdamar, L. \& Pedamallu, C. S., New Simulated Annealing Algorithms for Constrained Optimization

3 (2010) 347

Pan, T.-W., Hung, S.-W. \& Lu, W.-M., DEA

PerformanceMeasurement of the National Innovation System in Asia and Europe

3 (2010) 369

Pedamallu, C. S., see Özdamar, L.

Qi, L., see Zhang, Y.

3 (2010) 347

1 (2010) 55

Rangan, A. \& Tansu, A., Some Results on a New Class of Shock Models

$4(2010) 503$

Rehman, S. U. \& Asad, M. W. A., A Mixed-Integer Linear Programming (MILP) Model for Short-Range Production Scheduling of Cement Quarry Operations

Resing, J. A. C., see Artalejo, J. R.

Saha, S. \& Basu, M., Integrated Dynamic Pricing for Seasonal Products with Price and Time Dependent Demand

3 (2010) 315

3 (2010) 335

3 (2010) 393

2 (2010) 227

Sawaki, K., see Suzuki, A.

2 (2010) 189

Sedeño-Noda, A. \& González-Martín, C., ShortestPath Simplex Algorithm with a Multiple Pivot Rule: A Comparative Study

6 (2010) 677

Sharma, V., Dahiya, K. \& Verma, V., Capacitated Two-Stage

Time Minimization Transportation Problem

4 (2010) 457

Shayanfar, H. A., see Aghaei, J.

5 (2010) 587 
Shiraz, R. K., see Alirezaee, M. R.

5 (2010) 607

Singh, A., A Hybrid Permutation-Coded Evolutionary Algorithm forthe Early/Tardy Scheduling Problem

6 (2010) 713

Sobel, M. J., see Zhang, J.

Sun, W., Tian, N. \& Li, S., The Allocation of Customers in a Discrete-Time Multi-Server Queueing System

Sun, W., see Zhang, Y.

4 (2010) 477

6 (2010) 649

1 (2010) 55

Suzuki, A. \& Sawaki, K., The Valuation of Russian Options for Double Exponential Jump Diffusion Processes

$2(2010) 227$

Takano, Y. \& Yamamoto, Y., Metric-Preserving Reduction ofEarth Mover's Distance

$1(2010) 39$

Takashima, R., Goto, M. \& Tsujimura, M., Irreversible Investment, Operating Flexibility, and Time Lags

$2(2010) 271$

Takaya, Y. \& Konno, H., A Maximal Predictability Portfolio Subject to a Turnover Constraint

Tamba, Y., see Ito, D.

Tansu, A., see Rangan, A.

Tian, N., see Sun, W.

Tseng, T.-W., see Liao, S.-K.

Tsujimura, M., see Takashima, R.

Tu, B., see Li, Y.

Verma, V., see Sharma, V.

Vocaturo, F., see Beraldi, P.

Wang, H.-J. \& Ni, Q., A Convex Approximation Method for

Large Scale Linear Inequality Constrained Minimization

Wang, S., Zheng, L. \& Zhang, Z., Decomposition Algorithms for the Interval Scheduling Problem

Wang, W.-W., He, L.-M. \& Cheng, M.-B., Two-Stage Flowshop Scheduling Problems with Identical and Batch Processors

Wee, H.-M., see Yu, J. C. P.

Widyadana, G. A., see Yu, J. C. P.

$\mathrm{Xu}, \mathrm{H} .$, Sample Average Approximation Methods for a Class of Stochastic Variational Inequality Problems

1 (2010) 1

2 (2010) 243

4 (2010) 503

6 (2010) 649

6 (2010) 753

2 (2010) 271

3 (2010) 411

4 (2010) 457

3 (2010) 301

1 (2010) 85

4 (2010) 517

5 (2010) 617

4 (2010) 437

4 (2010) 437

Yagi, K. \& Sawaki, K., The Valuation of Callable-Puttable

Reverse Convertible Bonds

Yagiura, M., see Nishihara, M.

1 (2010) 103

2 (2010) 189

2 (2010) 211

2 (2010) 143

Yamada, T., see Kunita, H.

Yamamoto, Y., see Takano, Y.

1 (2010) 39

Yu, J. C. P., Wee, H.-M., Widyadana, G. A. \& Chang, J.-Y., The Effects of Inflation and Time Value of Money on a Production Model with a Random Product Life Cycle

4 (2010) 437 
Yu, J. C. P., Optimal Deteriorating Items Inventory Model with a Three-echelon Supply Chain Strategic Alliance

6 (2010) 693

Yuan, J., see He, C.

5 (2010) 577

Zhang, J. \& Sobel, M. J., Financially Optimal Inventory Policies with Non-Linear Replenishment Costs

$4(2010) 477$

Zhang, J., see Li, Y.

Zhang, S.-X., Cao, Z.-G. \& Zhang, Y.-Z., Scheduling with Discretely Compressible Release Dates to Minimize Makespan

5 (2010) 629

$4(2010) 493$

Zhang, Y., Sun, W. \& Qi, L., A Nonmonotone Filter Barzilai-Borwein Method for Optimization

1 (2010) 55

Zhang, Y.-Z., see Zhang, S.-X.

4 (2010) 493

Zhang, Z., see Wang, S.

4 (2010) 517

Zheng, L., see Wang, S.

4 (2010) 517

Zhou, X., see Lin, L.

6 (2010) 669 